\title{
A goal programming model that ensures efficient usage of limited interdiction budget in the procurement game
}

\author{
Gökhan Özçelik ${ }^{1, *}$ and Cevriye Gencer ${ }^{2}$ \\ ${ }^{1}$ Faculty of Engineering, Karadeniz Technical University \\ 06180 Ortahisar/Trabzon, Turkey \\ E-mail: < gozcelik@ktu.edu.tr> \\ ${ }^{2}$ Faculty of Engineering, Gazi University \\ 06570 Maltepe/Ankara, Turkey \\ E-mail: <ctemel@gazi.edu.tr>
}

\begin{abstract}
The paper investigates a procurement game between two rival firms considered as a network interdiction problem (NIP) where an interdictor, using limited interdiction budget, interdicts the node of supplier(s) on a capacitated single echelon multi products supply chain and tries to explicitly maximize the minimum procurement cost of a defender. In the study, a goal programming model is proposed to prevent the situations where the budget is used unnecessary (wasted budget) out of discrete interdiction variable.
\end{abstract}

Keywords: $\quad$ supplier interdiction, procurement game, network interdiction, integer programming

Received: July 27, 2017; accepted: July 19, 2018; available online: July 24, 2018

DOI: $10.17535 /$ crorr.2018.0007

\section{Introduction and background}

In this study, we consider a network interdiction approach where the main logic is based on game theory and analyze the competitive procurement process of two rival firms which are called leader and follower. These firms operate in the same activity area and need same materials. Because they need same materials for their production processes, the probability of contracting to the same suppliers is high. This situation may put the production levels of each other in danger due to the reduced amount of materials in suppliers. These risks encourage the firms to seek various policies for surviving in this competitive environment.

The leader firm wants to maximize the minimum cost that is achieved by the follower based on a limited interdiction budget for his own benefits (such as "to improve the competitiveness, to relatively reduce the costs"). It is assumed that the firms have sufficient information about each other. The procurement game is performed sequentially. Firstly, one of the firms (leader) interdicts one or more supplier's capacity within a given interdiction budget in order to maximize the minimum multi-product procurement cost that will be achieved by

\footnotetext{
* Corresponding author. 
another firm (follower). Later, the follower satisfies its own demand from remaining capacity. This problem is modeled as a bi-level mixed integer program and is converted into mixed integer program where the leader explicitly maximizes the minimum cost (achieved by the follower) by interdicting the supplier's capacity. However, unnecessary budget usage (wasted budget) occurs out of discrete interdiction. The main goal is to ensure the usage of the limited interdiction budget efficiently for the leader' interdiction plans. For this purpose, in the next subsections, firstly, the exact formulations for the leader and follower's problems are presented. Later, a goal programming approach that helps the leader to determine the most reasonable interdiction budget in accordance with own goals is proposed.

This leader-follower relationship is similar to static Stackelberg game that is firstly introduced in [16] and such a game can be presented mathematically as a bi-level programming problem. When we look at first studies on NIPs, we face with efforts to determine the most vital arc (link) or arcs on the network $[3,5,6,8,9,12,17,18]$. In these studies, the answer has been searched mainly for the question "Which components (arc or node) that disrupt the network at maximum level should be removed?" When the literature is investigated, we can say that the studies can be classified in two main headings as maximizing of the shortest path or the minimizing the maximum flow. Moreover, NIP framework has been still studying in many different scopes, namely; nuclear smuggling interdiction; national defense; sensor locations; facility interdiction; project interdiction etc. The other remarkable contributions are $[2,4,7,10,11,13,14,15,20,21]$.

It can be said that almost all studies prior to Wood [19] are specific to the application and are not extendible to more general contexts. Wood [19] is the first one adopted mathematical programming methods. He developed a min-max formulation of maximum flow network interdiction problem $(M F N I P)$ and then converted it to an integer-programming model [1]. In our study, the proposed goal programming model is developed based on Wood's [19] study.

The remainder of this study is organized as follows. In section 2, the problem is described and the exact formulations for the leader and follower's problems and a goal programming approach that is to help the leader to take strategic decisions related to the interdiction budget are presented. In section 3, computational studies are conducted. Finally, the study is concluded in section 4 .

\section{Problem formulations}

Consider a capacitated and directed network $G=(N, A)$ where the node set is $N$ and the arc set is $A$. In this network, the flow can move from the set of the suppliers $I \subset N$ to the set of the firms $F \subset N$. This flow is restricted by a positive integral capacity $S_{i}$. In the formulations, $i \in I$ and $p \in P$ are indices that belong to the suppliers and products, respectively. $\left(c_{i p}\right)$ is the procurement cost of the product $p$ that is procured from $i^{\text {th }}$ supplier. $\left(S_{i p}\right)$ is the capacity of product $p$ in $i^{\text {th }}$ supplier. $D_{p}^{F}$ is the demand for product $p$ of the follower. 


\subsection{Model-BI: The bi-level model of the leader's problem}

In this subsection, firstly the leader's problem is modeled as a bi-level, max-min program. The leader's decision variable $\left(y_{i}\right)$ takes the value of 1 if $i^{\text {th }}$ supplier is interdicted and 0 otherwise. The main goal of the leader is to maximize the minimum cost of the follower by interdicting the supplier(s). In Model II-BI, the objective function (1) maximizes the minimum cost of the follower, Constraints (2) are related to the suppliers' nominal capacities. These constraints restrict the amount of moved material on arc. Moreover, if $y_{i}=1$, it means that the leader interdicts $i^{\text {th }}$ supplier, that is, the capacity of $i^{\text {th }}$ supplier equals to zero, thus the follower does not procure material from this supplier any more. Similarly, if $y_{i}=0$, the leader does not interdict $i^{\text {th }}$ supplier and the capacity of $i^{\text {th }}$ supplier does not change for the follower's procurement process. Constraints (3) satisfy the demand of the follower. Constraints (4) are non-negativity constraints and constraints (5) restrict the number of interdiction based on interdiction budget and require interdiction variables to be binary where the interdiction cost of $i^{\text {th }}$ supplier is $r_{i}$.

$$
z^{*}=\max _{y} \min _{x} \sum_{i \in I} \sum_{p \in P} c_{i p} x_{i p}
$$

s.t.

$$
\begin{gathered}
x_{i p} \leq S_{i p}\left(1-y_{i}\right), \forall i \in I, \forall p \in P: \phi_{i p} \\
\sum_{i \in I} x_{i p} \geq D_{p}^{F}, \forall p \in P: \beta_{p} \\
x_{i p} \geq 0, \forall i \in I, \forall p \in P
\end{gathered}
$$

where,

$$
Y=\left\{y_{i} \in\{0,1\}: \sum_{i \in I} r_{i} y_{i} \leq R, R \in Z^{+}\right\}
$$

It is clear that for fixed $y_{i}$, the inner minimization is the follower's procurement model in Model II. In the next subsection, Model-BI is converted to nonlinear mixed integer program. Since this problem is unimodular, a mixed integer nonlinear "max-max" model is obtained by taking the dual of the inner minimization by fixing temporarily and then relaxing $y_{i}[19]$.

\subsubsection{Model-MINLP: Model-BI formulation as a mixed integer nonlinear program and its linearization}

With the above-mentioned transformation (taking dual form of the inner minimization problem), both objective functions are maximizations over differing sets of variables (the leader's primary variables, and the follower's dual variables). Thus, the following nonlinear mixed integer model is obtained. 


$$
z^{*}=\max _{y} \max _{\phi, \beta} \sum_{i \in I} \sum_{p \in P} S_{i p} \phi_{i p}\left(1-y_{i}\right)+\sum_{p \in P} D_{p}^{F} \beta_{p}
$$

s.t.

$$
\begin{aligned}
& \phi_{i p}+\beta_{p} \leq c_{i p}, \forall i \in I, \forall p \in P \\
& \sum_{i \in I} r_{i} y_{i} \leq R \\
& \phi_{i p} \leq 0, \forall i \in I, \forall p \in P \\
& \beta_{p} \geq 0, \forall p \in P \\
& y_{i} \in\{0,1\}, \forall i \in I
\end{aligned}
$$

\subsubsection{Model-F: Final model of the leader's problem}

For the final model of the leader's problem, Model-MINLP is linearized by adding some constraints to the model. It is known that $\phi_{i p} \leq 0$ and $y_{i} \in\{0,1\}$. The Model-MINLP can be linearized with the help of these restrictions and it does not cause a loss of optimality.

Let us replace $\left(1-y_{i}\right) \phi_{i p}$ with $\mu_{i p} \leq 0$ and add the set of constraints (14) $\mu_{i p} \leq \phi_{i p}+y_{i} M$ where $M$ is a big enough number. This yields the following mixed integer programming (MIP). If $y_{i}=1$ in an optimal solution, $S_{i p}\left(1-y_{i}\right) \phi_{i p}$ is equal to zero. Similarly, if $y_{i}=0$ in an optimal solution, $S_{i p}\left(1-y_{i}\right) \phi_{i p}$ is equal to $S_{i p} \phi_{i p}$. It is known that we replace $S_{i p}\left(1-y_{i}\right) \phi_{i p}$ with $S_{i p} \mu_{i p}$. Now, let us work the linearization, if $y_{i}=1$ in any optimal solution, $\mu_{i p}$ must equal to 0 . For maximizing problem, constraints (14) and (18) are satisfied for $\mu_{i p} \leq \phi_{i p}+y_{i} M$ and $\mu_{i p} \leq 0$. As, constraints (14) have big right hand side values, when the constraints (14) and (18) are considered, simultaneously, $\mu_{i p}$ equals to0. Furthermore, if $y_{i}=0$ in any optimal solution, $\mu_{i p}$ must equal to $\phi_{i p}$. Constraints (14) and (18) are satisfied for $\mu_{i p} \leq \phi_{i p}+0 M$ and $\mu_{i p} \leq 0$. Due to the maximizing objective function (12), when the constraints (14) and (18) are considered, simultaneously, it must be true that $\mu_{i p}=\phi_{i p}$. It is clear that this situation justifies the correctness of the linearization. Finally, Model-F gives an interdiction plan based on a specific interdiction budget to the leader.

$$
z^{*}=\max _{y, \mu, \phi, \beta} \sum_{i \in I} \sum_{p \in P} S_{i p} \mu_{i p}+\sum_{p \in P} D_{p}^{F} \beta_{p}
$$

s.t.

$$
\phi_{i p}+\beta_{p} \leq c_{i p}, \forall i \in I, \forall p \in P
$$




$$
\begin{aligned}
& \mu_{i p} \leq \phi_{i p}+y_{i} M^{*}, \forall i \in I, \forall p \in P \\
& \sum_{i \in I} r_{i} y_{i} \leq R \\
& \phi_{i p} \leq 0, \forall i \in I, \forall p \in P \\
& \beta_{p} \geq 0, \forall p \in P \\
& \mu_{i p} \leq 0, \forall i \in I, \forall p \in P \\
& y_{i} \in\{0,1\}, \forall i \in I
\end{aligned}
$$

\subsection{Model-G: A goal programming approach for efficient decisions of the leader}

In this subsection, a weighted $\left(w_{1}, w_{2}\right)$ goal programming approach that allows the leader to use interdiction budget efficiently and to take right decisions is proposed. For this purpose, two main goals are considered for the leader. One of these is the damage goal $\left(G_{1}\right)$, namely, the leader does not want to fall below a certain damage that is given to the follower. $\left(F C_{\text {initial }}\right)$ is the cost of the follower when there is no interdiction.

Similarly, the other goal is related to the interdiction budget $\left(G_{2}\right)$. The leader does not want to ascend on a certain budget. The objective function (20) minimizes the deviations $\left(d_{1}^{-}, d_{2}^{+}\right)$from the goals. The goal constraints are defined in $(23,24)$.

$$
z^{* * *}=\min _{y, \mu, \phi, \beta, d_{1}^{-}, d_{1}^{+}, d_{2}^{-}, d_{2}^{+}} w_{1} d_{1}^{-}+w_{2} d_{2}^{+}
$$

s.t.

$$
\begin{gathered}
\phi_{i p}+\beta_{p} \leq c_{i p}, \forall i \in I, \forall p \in P \\
\mu_{i p} \leq \phi_{i p}+y_{i} M^{*}, \forall i \in I, \forall p \in P \\
\sum_{i \in I} \sum_{p \in P} S_{i p} \mu_{i p}+\sum_{p \in P} D_{p}^{F} \beta_{p}-F C_{\text {initial }}+d_{1}^{-}-d_{1}^{+}=G_{1} \\
\sum_{i \in I} r_{i} y_{i}+d_{1}^{-}-d_{1}^{+}=G_{2} \\
\phi_{i p} \leq 0, \forall i \in I, \forall p \in P \\
\beta_{p} \geq 0, \forall p \in P \\
\mu_{i p} \leq 0, \forall i \in I, \forall p \in P
\end{gathered}
$$




$$
\begin{aligned}
& y_{i} \in\{0,1\}, \forall i \in I \\
& d_{1}^{-}, d_{1}^{+}, d_{2}^{-}, d_{2}^{-} \geq 0
\end{aligned}
$$

Thanks to Model-G, it is aimed to facilitate the analysis of the leader based on different deviations weight. In fact, this analysis can be called as sensitivity analysis. It helps to investigate the value of the deviations based on weights and different parameters. In the next section, an illustrative study is conducted.

\section{Computational studies}

In this section, by conducting a numerical example, some analysis is made for the leader's interdiction plan. As mentioned-above, the leader has limited interdiction budget, and he should use this budget efficiently. As seen in Table 1, there is a "cost of interdiction" (cost of contract; namely, the amount of the money is given by the leader to the supplier not to sell material to the follower) for each supplier. If the leader does not interdict any supplier, the results of the procurement problem of the follower are as given in Table 2. On behalf of investigation the efficient usage of the interdiction budget, until the remaining capacity is insufficient for the follower, the changes in cost of the follower are investigated for each budget level $\left(R \in Z^{+}\right)$and are given Table 3 .

\begin{tabular}{|c|c|c|c|c|c|}
\hline \multirow{2}{*}{$\begin{array}{c}\# \text { of } \\
\text { Suppliers }\end{array}$} & \multicolumn{2}{|c|}{ Capacities (gallon) } & \multicolumn{3}{|c|}{ Costs $(\$ /$ unit $)$} \\
\cline { 2 - 6 } & $\begin{array}{c}\text { Suppliers } \\
\left(S_{i 1}\right)\end{array}$ & $\begin{array}{c}\text { Suppliers } \\
\left(S_{i 2}\right)\end{array}$ & $\begin{array}{c}\text { Procurement } \\
\left(c_{i 1}\right)\end{array}$ & $\begin{array}{c}\text { Procurement } \\
\left(c_{i 2}\right)\end{array}$ & $\begin{array}{c}\text { Interdiction } \\
(\text { Contract })\left(r_{i}\right)\end{array}$ \\
\hline 1. & $(50)$ & $(30)$ & 1 & 2 & 19 \\
\hline 2. & $(50)$ & $(30)$ & 2 & 2 & 12 \\
\hline 3. & $(60)$ & $(40)$ & 3 & 3 & 17 \\
\hline 4. & $(60)$ & $(40)$ & 4 & 5 & 11 \\
\hline 5. & $(70)$ & $(40)$ & 1 & 3 & 16 \\
\hline 6. & $(70)$ & $(45)$ & 2 & & \\
\hline
\end{tabular}

Note: $D_{1}^{F}=90, D_{2}^{F}=85$.

Table 1: Data of the problem.

In this section, all MIP formulations and algorithms are implemented in C\# using GAMS 23.5.1 (via CPLEX solver). All experiments were performed on a PC that have Intel(R) Core(TM) i7 CPU 2.00 GHz processors and 6.00 GB RAM.

Firstly, starting from $R=0$, the interdiction budget $(R)$ is increased gradually (see Table 3). If the optimal solution is an abnormally large number, there exists no optimal flow satisfying the follower's demand, and thus the dual problem of inner problem is unbounded. For this reason, the analysis is conducted up to $(R<56)$ (see Table 3$)$. 


\begin{tabular}{|c|c|c|}
\hline \multirow{2}{*}{ \# of Suppliers } & \multicolumn{2}{|c|}{ Amount of procured materials (gallon) } \\
\cline { 2 - 3 } & $\left(\mathrm{x}_{\mathrm{i} 1}\right)$ & Suppliers $\left(\mathrm{x}_{\mathrm{i} 2}\right)$ \\
\hline 1. & $(50)$ & $(30)$ \\
\hline 2. & $*$ & $(30)$ \\
\hline 3. & $*$ & $(25)$ \\
\hline 4. & $*$ & $*$ \\
\hline 5. & $*$ & $*$ \\
\hline 6. & $(40)$ & $*$ \\
\hline \multicolumn{2}{|c|}{ Total cost of the follower: $\$ 285$} \\
\hline
\end{tabular}

Table 2: The results of the procurement model of the follower when there is no interdiction.

\begin{tabular}{|c|c|c|c|c|c|c|c|c|c|c|c|c|c|c|c|}
\hline$R$ & $z^{*}$ & $R$ & $z^{*}$ & $R$ & $z^{*}$ & $R$ & $z^{*}$ & $R$ & $z^{*}$ & $R$ & $z^{*}$ & $R$ & $z^{*}$ & $R$ & $z^{*}$ \\
\hline 0 & $285^{\mathrm{a}}$ & 7 & 285 & 14 & 315 & 21 & 335 & 28 & 335 & 35 & 365 & 42 & 405 & 49 & 435 \\
\hline 1 & 285 & 8 & 285 & 15 & 315 & 22 & 335 & 29 & 335 & 36 & 365 & 43 & 405 & 50 & 435 \\
\hline 2 & 285 & 9 & 285 & 16 & 315 & 23 & 335 & 30 & 335 & 37 & 365 & 44 & 405 & 51 & 455 \\
\hline 3 & 285 & 10 & 285 & 17 & 315 & 24 & 335 & 31 & 365 & 38 & 365 & 45 & 405 & 52 & 455 \\
\hline 4 & 285 & 11 & 285 & 18 & 315 & 25 & 335 & 32 & 365 & 39 & 365 & 46 & 405 & 53 & 455 \\
\hline 5 & 285 & 12 & $315^{\mathrm{b}}$ & 19 & $335^{\mathrm{c}}$ & 26 & 335 & 33 & 365 & 40 & 405 & 47 & 435 & 54 & 455 \\
\hline 6 & 285 & 13 & 315 & 20 & 335 & 27 & 335 & 34 & 335 & 41 & 405 & 48 & 435 & 55 & 475 \\
\hline
\end{tabular}

Note: After $\mathrm{R}_{\max }=55$, the next interdictions are infeasible since the remaining capacity of any product in non-interdicted supplier(s) is insufficient for the follower.

Table 3: All solutions.

As seen in Table $(2,3)$, according to the results, the cost of the follower is $\$ 285^{(\mathrm{a})}$ when there is no interdiction. If the leader gives damage to the follower, he should determine the interdiction budget at least $\$ 12$. In this situation, the leader interdicts $2^{\text {nd }}$ supplier. Thus, the cost of the follower is $\$ 315^{(b)}$ and the follower suffers a loss as $\$ 30$. If the leader gives a little more damage to the follower, he should determine the interdiction budget at least $\$ 19$ since the cost of the follower remains the same up to $\$ 18$. In this situation, the leader interdicts $1^{\text {st }}$ supplier. Thus, the cost of the follower is $\$ 335^{(c)}$ and the follower suffers a loss as $\$ 50$. Similarly, when the leader wants to increase the damage that is given to the follower, he should track the critical budgets in Fig. 1, respectively. It is clear that the interdictions with exception of these budgets are unnecessary in terms of effective usage of the budget. So, the leader should investigate the cost of the follower on each budget level before take a decision. 


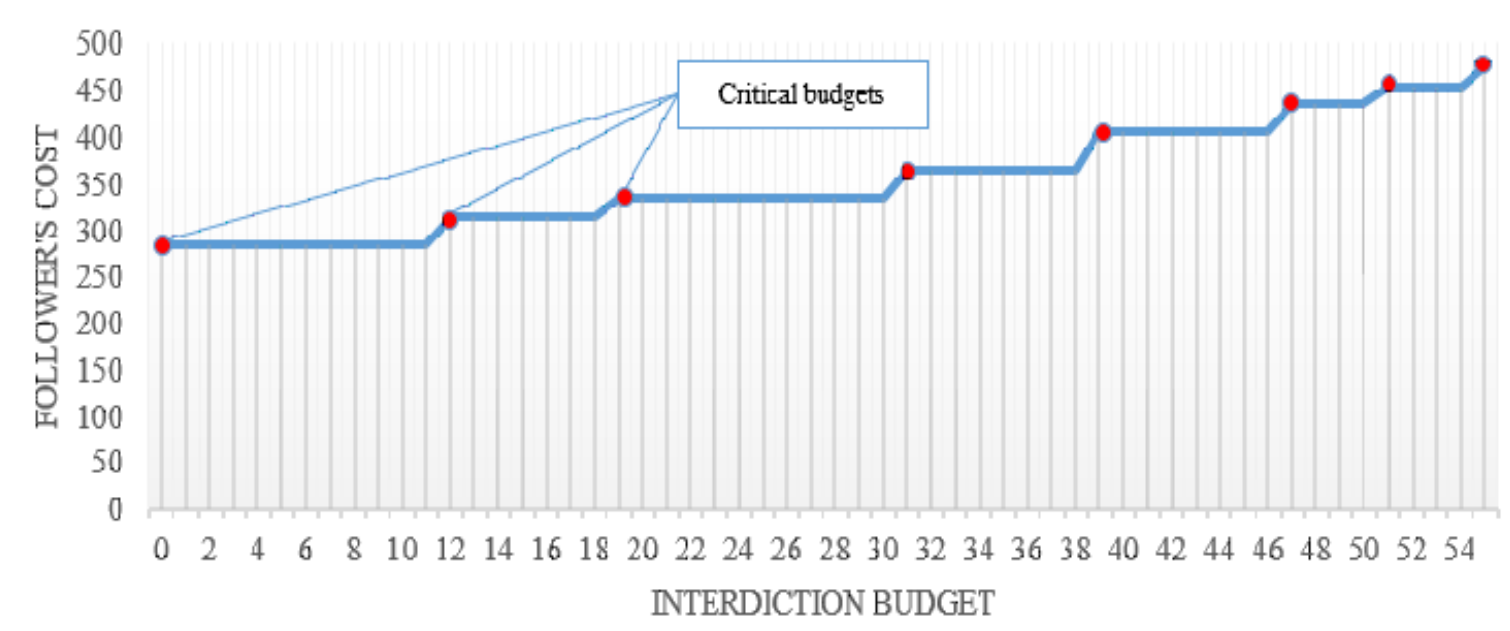

Figure 1: The graph of follower's cost $\mathcal{E}$ interdiction budget

In order to avoid these unnecessary interdictions, in the subsection 2.2, a goal programming that helps analysis of relationship between the budget and damage that is given to the follower is presented. The budget $\left(G_{1}\right)$ and the damage $\left(G_{2}\right)$ are considered as goals. The leader both does not want to fall below a certain damage $\left(d_{1}^{-}\right)$that is given to the follower and does not want to ascend on a certain budget $\left(d_{2}^{+}\right)$. The aim is to minimize the deviations $\left(d_{1}^{-}, d_{2}^{+}\right)$from the goals. In this regard, the different plans that are given below, are derived related to the weights of the goals and the results are shown in tables $(4,5)$ :

- Plan I: The usage of budget and damage that will give to the follower have equal importance $\left(w_{1}=w_{2}\right)$;

- $\quad$ Plan II: The approach is prone to use the budget efficiently $\left(w_{1}<w_{2}\right)$;

- $\quad$ Plan III: The approach is prone to give the more damage to the follower $\left(w_{1}>w_{2}\right)$.

Consider that the leader wants to fix the interdiction budget at $\$ 20\left(G_{2}=20\right)$. If the leader wants to give damage to the follower at least $\$ 90$, according to the Plan I and Plan III, he should increase the budget as $\$ 19$. However, if the budget constraint is more important than the damage (Plan II), he should increase the budget as $\$ 11$. In this situation, he fails to reach the damage goal $(\$ 90)$. He can give damage to the follower as $\$ 80$ with an interdiction budget of $\$ 31$. Similarly, the values of the deviations according to the different plans are analyzed up to the maximum damage $(475-285=\$ 190)$ that can be given to the follower and the results are given in Table 4. 


\begin{tabular}{|c|c|c|c|c|c|c|c|c|c|c|c|c|}
\hline \multirow{3}{*}{$\begin{array}{c}G_{2}=20 \\
G_{1}\end{array}$} & \multicolumn{4}{|c|}{ Plan I } & \multicolumn{4}{|c|}{ Plan II } & \multicolumn{4}{|c|}{ Plan III } \\
\hline & \multicolumn{4}{|c|}{$w_{1}: 0.5, w_{2}: 0.5$} & \multicolumn{4}{|c|}{$w_{1}: 0.3, w_{2}: 0.7$} & \multicolumn{4}{|c|}{$w_{1}: 0.7, w_{2}: 0.3$} \\
\hline & $d_{1}^{-}$ & $d_{1}^{+}$ & $d_{2}^{-}$ & $d_{2}^{+}$ & $d_{1}^{-}$ & $d_{1}^{+}$ & $d_{2}^{-}$ & $d_{2}^{+}$ & $d_{1}^{-}$ & $d_{1}^{+}$ & $d_{2}^{-}$ & $d_{2}^{+}$ \\
\hline 50 & 0.00 & 0.00 & 1.00 & 0.00 & 0.00 & 0.00 & 1.00 & 0.00 & 0.00 & 0.00 & 1.00 & 0.00 \\
\hline 55 & 5.00 & 0.00 & 1.00 & 0.00 & 5.00 & 0.00 & 1.00 & 0.00 & 0.00 & 0.00 & 0.00 & 11.00 \\
\hline 60 & 10.00 & 0.00 & 1.00 & & 10.00 & 0.00 & & & & 0.00 & 0.00 & 11.00 \\
\hline 65 & & 0.00 & & & & 0.00 & & & & 0.00 & 0.00 & 11.00 \\
\hline 70 & & 0.00 & & & & & & & & & & 11.00 \\
\hline 75 & 00 & 0.00 & 0.00 & 11.00 & 25.00 & 0.00 & 1.00 & 0.00 & .00 & 0.00 & 0.00 & 11.00 \\
\hline 80 & & & & & & & & & & & & \\
\hline 85 & & 0.00 & & 11 & & 0.00 & & 00 & & 00 & 00 & 9.00 \\
\hline 90 & & & & & & 0.00 & & & & 00 & & 9.00 \\
\hline 95 & & & & & & & & & & & & \\
\hline 100 & & & & & & & & & & & & \\
\hline 105 & & 0.00 & & & & & & & & & & 19.00 \\
\hline 110 & & 0.00 & & 19.00 & & 0.00 & & 19.00 & 0.00 & 0.00 & 0.00 & 19.00 \\
\hline 115 & & 0.00 & & 19.00 & & 0.00 & & & & 0.00 & 0.00 & 19.00 \\
\hline 120 & & 0.00 & 0.0 & 19.00 & & 0.00 & & 19.00 & 0.00 & 0.00 & 0.00 & 19.00 \\
\hline & & & & & & & & & & & & \\
\hline 130 & & & & & & & & & & & & 27.00 \\
\hline 135 & & & & & & & & & & & & \\
\hline 140 & & 0.00 & 0.1 & 27.00 & & 0.00 & 0.0 & 27.00 & 0.00 & 0.00 & 0.00 & 27.00 \\
\hline 145 & & & & & & & & & & & & \\
\hline 150 & & & & & & & & & & & & \\
\hline 155 & & & & & & & & & & 0.00 & 0.00 & 31.00 \\
\hline 160 & & & & & & & & & & 0.00 & & 31.00 \\
\hline 165 & 0.00 & 0.00 & 0.00 & 31.00 & 0.00 & 0.00 & 0.00 & 31.00 & 0.00 & 0.00 & 0.00 & 31.00 \\
\hline 170 & & & & & & & & & & & & \\
\hline 175 & & 0.00 & 0.00 & 35.00 & 5.00 & 0.00 & 0.00 & 31.00 & 0.00 & 0.00 & 0.00 & 35.00 \\
\hline 180 & & 0.00 & & 35.00 & 0.00 & 0.00 & 0.00 & 35.00 & 0.00 & 0.00 & 0.00 & 35.00 \\
\hline 185 & 0.00 & 0.00 & 0.00 & 35.00 & 0.00 & 0.00 & 0.00 & 35.00 & 0.00 & 0.00 & 0.00 & 35.00 \\
\hline $190^{*}$ & 0.00 & 0.00 & 0.00 & 35.00 & 0.00 & 0.00 & 0.00 & 35.00 & 0.00 & 0.00 & 0.00 & 35.00 \\
\hline
\end{tabular}

Note: The analysis is conducted up to the maximum damage that can be given to the follower.

Table 4: The values of the deviations according to the different plans with respect to $G_{2}=20$ 


\begin{tabular}{|c|c|c|c|c|c|c|c|c|c|c|c|c|}
\hline \multirow{2}{*}{$G_{1}=150$} & \multicolumn{4}{|c|}{ Plan I } & \multicolumn{4}{c|}{ Plan II } & \multicolumn{5}{c|}{ Plan III } \\
\cline { 2 - 13 } & \multicolumn{3}{|c|}{$w_{1}: 0.5, w_{2}: 0.5$} & \multicolumn{4}{c|}{$w_{1}: 0.2, w_{2}: 0.8$} & \multicolumn{4}{c|}{$w_{1}: 0.8, w_{2}: 0.2$} \\
\hline$G_{2}$ & $d_{1}^{-}$ & $d_{1}^{+}$ & $d_{2}^{-}$ & $d_{2}^{+}$ & $d_{1}^{-}$ & $d_{1}^{+}$ & $d_{2}^{-}$ & $d_{2}^{+}$ & $d_{1}^{-}$ & $d_{1}^{+}$ & $d_{2}^{-}$ & $d_{2}^{+}$ \\
\hline 10 & 0.00 & 0.00 & 0.00 & 37.00 & 120.00 & 0.00 & 0.00 & 2.00 & 0.00 & 0.00 & 0.00 & 37.00 \\
\hline 15 & 0.00 & 0.00 & 0.00 & 32.00 & 100.00 & 0.00 & 0.00 & 4.00 & 0.00 & 0.00 & 0.00 & 32.00 \\
\hline 20 & 0.00 & 0.00 & 0.00 & 27.00 & 100.00 & 0.00 & 1.00 & 0.00 & 0.00 & 0.00 & 0.00 & 27.00 \\
\hline 25 & 0.00 & 0.00 & 0.00 & 22.00 & 30.00 & 0.00 & 0.00 & 14.00 & 0.00 & 0.00 & 0.00 & 22.00 \\
\hline 30 & 0.00 & 0.00 & 0.00 & 17.00 & 30.00 & 0.00 & 0.00 & 9.00 & 0.00 & 0.00 & 0.00 & 17.00 \\
\hline 35 & 0.00 & 0.00 & 0.00 & 12.00 & 30.00 & 0.00 & 0.00 & 4.00 & 0.00 & 0.00 & 0.00 & 12.00 \\
\hline 40 & 0.00 & 0.00 & 0.00 & 7.00 & 0.00 & 0.00 & 0.00 & 7.00 & 0.00 & 0.00 & 0.00 & 7.00 \\
\hline 45 & 0.00 & 0.00 & 0.00 & 2.00 & 0.00 & 0.00 & 0.00 & 2.00 & 0.00 & 0.00 & 0.00 & 2.00 \\
\hline 50 & 0.00 & 0.00 & 3.00 & 0.00 & 0.00 & 0.00 & 3.00 & 0.00 & 0.00 & 0.00 & 3.00 & 0.00 \\
\hline 55 & 0.00 & 0.00 & 4.00 & 0.00 & 0.00 & 0.00 & 4.00 & 0.00 & 0.00 & 0.00 & 4.00 & 0.00 \\
\hline
\end{tabular}

Note: $\mathrm{R}_{\max }=55$, then the analysis is stopped.

Table 5: The values of the deviations according to the different plans with respect to $G_{1}=150$

This analysis can be made from other side (in terms of the interdiction budget), as well. Similarly, it can be considered that the leader wants to fix the damage that is given to the follower at $\$ 150\left(G_{1}=150\right)$ (See Table 5$)$. If the leader wants to determine the interdiction budget at most $\$ 30$, according to the Plan I and Plan III, he should increase the budget as $\$ 17$. However, if the budget constraint is more importance than the damage (Plan II), he should increase the budget as $\$ 9$. To clarify, he fails to reach the damage goal $(\$ 150)$. He can give damage to the follower as $\$ 120$ with an interdiction budget of $\$ 39$. In these analyses, the numerical values are increased five by five, but the more sensitive analysis can be conducted by increasing the numerical values one by one, as well.

\section{Conclusion}

In this paper, we study the bi-level procurement game (Model-BI) that can be modeled as a static Stackelberg game between a leader and a follower. Then, it is converted into mixed integer program (Model-F) where the leader explicitly maximizes the minimum cost (achieved by the follower) with interdicting the supplier. The aim is to investigate the relationship between the interdiction budget of the leader and damage that is given to the follower. According to the results in Table 3, it is seen that there are unnecessary interdictions in terms of effective usage of the budget. So, in order to avoid these unnecessary interdictions, a goal programming (Model-G) that both helps the determination of relationship between the budget and damage that is given to the follower and allows to take efficient decisions about the interdiction is presented. Computational results indicate that Model-G is a powerful tool in terms of adjustment of the budget and damage for the leader. 


\section{References}

[1] Akgün, İ., Tansel, B. Ç. and Wood, R. K. (2011). The multi-terminal maximum-flow network-interdiction problem. European Journal of Operational Research, 211(2), 241-251.

[2] Aksen, D., S, engül Akca, S. and Aras, N. (2014). A bilevel partial interdiction problem with capacitated facilities and demand outsourcing. Computers \& Operations Research, 41, 346-358.

[3] Ball, M. O., Golden, B. L. and Vohra, R. V. (1989). Finding the most vital arcs in a network. Operations Research Letters, 8(2), 73-76.

[4] Church, R. L. and Scaparra, M. P. (2007). Protecting critical assets: the r-interdiction median problem with fortification. Geographical Analysis, 39(2), 129-146.

[5] Corley, H. and Chang, H. (1974). Finding the $\mathrm{n}$ most vital nodes in a flow network. Manage Science, 21(3), 362-364.

[6] Corley, H. and David, Y. S. (1982). Most vital links and nodes in weighted networks. Operations Research Letters, 1(4), 157-160.

[7] Kennedy, K. T., Deckro, R. F., Moore, J. T. and Hopkinson, K. M. (2011). Nodal interdiction. Mathematical and Computer Modelling, 54(11-12), 3116-3125. ichepi

[8] Lubore, S., Ratliff, H. and Sicilia, G. T. (1971). Determining the most vital link in a flow network. Naval Research Logistics Quarterly, 18(4), 497-502.

[9] Malik, K., Mittal, A. K. and Gupta, S. K. (1989). The k most vital arcs in the shortest path problem. Operations Research Letters, 8(4), 223-227.

[10] Morton, D. P., Pan, F. and Saeger, K. J. (2007). Models for nuclear smuggling interdiction. IIE Transactions, 39(1), 3-14.

[11] Prince, M., Smith, J. C. and Geunes, J. (2013). A three-stage procurement optimization problem under uncertainty. Naval Research Logistics (NRL), 60(5), 395-412.

[12] Ratliff, H. D., Sicilia, G. T. and Lubore, S. H. (1975). Finding the most vital links in flow networks. Management Science, 21(5), 531-539.

[13] Romich, A., Lan, G. and Smith, J. C. (2015). Algorithms for optimizing the placement of stationary monitors. IIE Transactions (Institute of Industrial Engineers), 47(6), 556-576.

[14] Salmeron, J., Wood, K. and Baldick, R. (2009). Worst-case interdiction analysis of largescale electric power grids. IEEE Transactions on Power Systems, 24(1), 96-104.

[15] Shen, S., Smith, J. C. and Goli, R. (2012). Exact interdiction models and algorithms for disconnecting networks via node deletions. Discrete Optimization, 9(3), 172-188.

[16] Stackelberg, H. (1952). The theory of the market economy. London: William Hodge \& Co.

[17] Wollmer, R. (1963). Some methods for determining the most vital link in a railway network. Santa Monica, California: Rand Corporation.

[18] Wollmer, R. (1964). Removing Arcs from a Network. Operations Research, 12(6), 934-940.

[19] Wood, R. K. (1993). Deterministic network interdiction. Mathematical and Computer Modelling, 17(2), 1-18.

[20] Zenklusen, R. (2010). Matching interdiction. Discrete Applied Mathematics, 158(15), 1676-1690.

[21] Zhang, P. and Fan, N. (2017). Analysis of budget for interdiction on multicommodity network flows. Journal of Global Optimization, 67(3), 495-525. 